\title{
Nickel-titanium pseudo-elastic behavior under equi-biaxial dynamic loading conditions
}

\author{
Pierre Quillery ${ }^{1, *}$, Bastien Durand ${ }^{1}$, Olivier Hubert ${ }^{1}$, and Han Zhao ${ }^{1,2}$ \\ ${ }^{1}$ LMT, ENS Paris-Saclay / Université Paris-Saclay / CNRS, 61 avenue du Président Wilson 94230 Cachan, France \\ ${ }^{2}$ UFR Ingénierie, Université Pierre et Marie Curie, Sorbonne Universités, Paris
}

\begin{abstract}
.
Shape memory alloys (SMA) undergo a solid-solid phase transformation called martensitic transformation, involving a "high temperature" phase (austenite) and a "low temperature" phase (martensite). The stress-strain pseudo-elastic behavior of a nickel-titanium under equi-biaxial dynamic compression is measured thanks to a new home-made impact testing set-up using split Hopkinson bars. The use of thermal and optical cameras allows strain and heating sources fields to be identified. The stress field is estimated by the combination of the strain gauges information placed on bars, and a finite element analysis of the specimen. Experimental average stress-strain behavior and thermal emission are finally compared to the results of a finite difference axisymmetric model where the constitutive law is given by a fully coupled stochastic multi scale model.
\end{abstract}

\section{Introduction}

The solid-solid phase transformation can be activated in SMA by a thermal loading (heating or cooling) or a mechanical loading (stress) and explains for example the pseudo-elastic phenomenon where high reversible deformations $(>6 \%)$ can be reached during a tensile loading. Although the uni-axial dynamic pseudo elastic behavior of SMA is relatively well documented today, behavior under multiaxial stress remains unknown. Such knowledge is however essential for the validation of multi-axial models, and finally for the democratization of these materials in current applications. SMA are subject to complex stress states, due to thermal and mechanical loadings and due to their geometries. For super-elasticity as well as shape memory effect, the nickel-titanium behavior is non-linear and stress states are multi-axial. The description of first order austenite to martensite phase transformation is usually enough to model uni-axial behaviors [1]. A multiscale description of the material under multi-axial loadings is however necessary to determine reliable 3D models. In addition, the thermal aspects can not be neglected because they govern the shape memory effect. Indeed, thermal effects are preponderant during a dynamic adiabatic loading. In this paper, thermal and kinematic fields measurements are used to observe the austenite to martensite phase transformation in biaxial condition. Experiments are compared to the results of a finite difference axisymmetric model where the constitutive law is given by a fully coupled stochastic multi scale model. The first part is dedicated to the presentation of the experimental set-up and protocol, then experimental results are presented and compared to simulation,

\footnotetext{
*e-mail: quillery@1mt.ens-cachan.fr
}

highlighting some dynamic effects not considered in the present modeling.

\section{Experimental set-up}

An home-made biaxial set-up coupled with a split Hopkinson system is used to submit the specimen to an equibiaxial loading. Hopkinson systems allow high strain rates, about $10 / s$ to $5000 / s$ to be reached $[3,5]$. The constitutive material of the bars is known and used in the field of linear elasticity, so that the system can be considered as a way of instrumentation. Strain gauges make it possible to determine the strain waves propagating in the bar and thus to evaluate forces and velocities conditions at the interfaces between bars and specimen. In the biaxial setup (see figure 1), the impact is carried out by a single input bar, via a return angle system and two outgoing bars, so that the sample is loaded along two orthogonal directions. The angle deflection mechanism works thanks to two planes inclined at $45^{\circ}$ relatively to the axis of the bar and allowing orthogonal compression.

The Hopkinson formulae [3] firstly make it possible to determine the two orthogonal forces. We seek to determine the transition matrix from forces to stresses using equation (1), where $m_{i j}$ parameters have to be identified using a finite element elastic modeling of the specimen structure.

$$
\left(\overline{\overline{\sigma_{x x}}}\right)=\left(\begin{array}{ll}
m_{x x} & m_{x y} \\
m_{y x} & m_{y y}
\end{array}\right)\left(\begin{array}{l}
F_{x} \\
F_{y}
\end{array}\right)
$$

Gauges are glued on incident and on the two transmitter bars to measure and calculate the strains thanks to usual Wheatstone bridge facilities (recording frequency is 


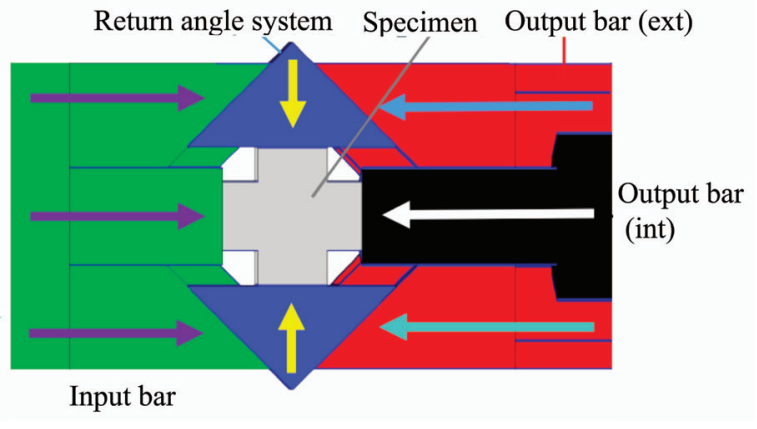

Fig. 1. Set-up principle for dynamic equibiaxial compression test.

$500 \mathrm{KHz}$ ). The equibiaxial behavior is investigated in the study (angles of return angle system is fixed at $45^{\circ}$ ). The cruciform specimen is designed to concentrate stress in the central area (region of interest - ROI) and avoid buckling. The ROI is a $3 \mathrm{~mm}$ diameter circle with a thickness of $0.5 \mathrm{~mm}$ especially machined in the center of a $2 \mathrm{~mm}$ thick specimen. The maximum overall dimension is fixed by the Hopkinson bar system and the biaxial set-up. External dimension of specimen are $8 \mathrm{~mm} \times 8 \mathrm{~mm}$, the cruciform geometry is shown in figure $2 \mathrm{a}$.

During the test, the biaxial specimen is tracked by a high speed numerical camera to accurately calculate its strain field by digital image correlation (DIC). The recording is realized by a SA5 fast-cam at $50000 \mathrm{~Hz}$ and on a $512 \mathrm{px} \times 271 \mathrm{px}$ area. The whole specimen surface is recorded to evaluate the free-body motions due to the Hopkinson bar system (kinematic boundary conditions). DIC principle consists to calculate the difference between two images, a reference one and a distorted one. An image is seen numerically as a function characterizing the gray level of each pixel. If we call $f(x)$ the function of the reference image and $g(x)$ the function of the deformed image, the determination of the displacement field $u(x)$ is obtained by minimizing equation (2).

$$
g(x+u(x))=f(x)
$$

Correlation code (Correli-RT3) has been used considering the displacement field as continuous. This code allows the identification of displacement on each node of a finite element triangular mesh. The obtained displacement is regularized by an elastic solution [4]. A thermal camera placed in front of the other side of sample which has been painted in black allows the temperature field to be measured. A calibration of the infrared sensor with a black body allows the link between the digital level (function of electromagnetic radiation and emissivity) measured by the camera and the temperature to be obtained. Figure $2 \mathrm{~b}$ shows the full range calibration and the restricted area chosen to increase the recording frequency to $15000 \mathrm{~Hz}$. This observation area, of about $64 \times 8$ pixels, enables to track the specimen during the testing and to compensate the free body displacement.

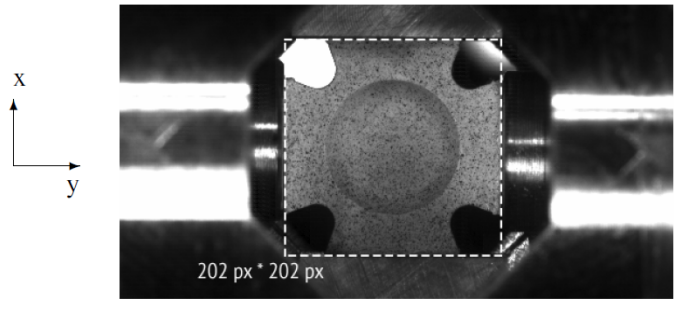

(a) Reference image and area for DIC

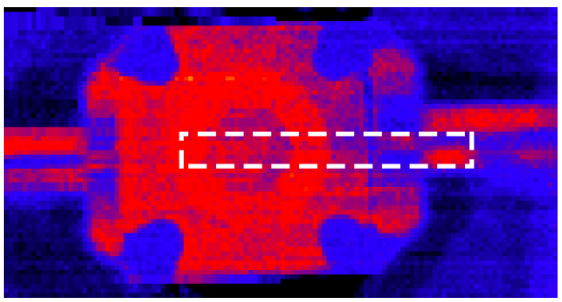

(b) Observation zone for a $15000 \mathrm{~Hz}$ infrared recording $(64 p x \times 8 p x)$

Fig. 2. Optical and thermal image settings.

\section{Experimental results}

Figure 3a shows the strain measured by the gauges as function of time. After time shifting to virtually transport the signals from the strain gauges to the multi-axial set-up interfaces, and after Hopkinson formulae application, we can plot the forces and the velocities at these interfaces (figures $3 \mathrm{~b}$ and 3c). During the steady state, all velocities are equal, and the forces applied by the incident and the transmitted bars are equal too. In our case, we have to compare the incident bar force to the sum of the two transmission bars forces to check at the equilibrium [6]. These requirements are confirmed in figure 3c. However we observe that force in the internal transmitter bar is a few lower than the force in the external transmitter bar denoting that biaxial loading is not perfectly equiproportional. This difference can be explained by a friction in the biaxial set up, higher along $x$ direction than along $y$ direction. Average stress components $\sigma_{x x}$ and $\sigma_{y y}$ are calculated form forces thanks to equation 1 (parameters used are: $m_{x x}=m_{y y}=0.2941 \mathrm{~mm}^{-2}$ and $\left.m_{x y}=m_{y x}=-0.0794 \mathrm{~mm}^{-2}\right)$. Strain components $\epsilon_{x x}, \epsilon_{y y}$ and $\epsilon_{x y}$ are calculated in the ROI from DIC measurements. $\epsilon_{x x}$ and $\epsilon_{y y}$ are plotted as function of time in figure 4a. Strains do not seem perfectly synchronized. The lower magnitude of $\epsilon_{y y}$ comparing to $\epsilon_{x x}$ is in accordance with the lower stress level along $y$ axis. At the beginning, the strain along $y$ can be related to a Poisson effect. At the end, the strain is reaching about $3.5 \%$. The infrared observations allow us to calculate the temperature increase during the test. Figure 5 illustrates this brutal increase during the first $1 \mathrm{~ms}$. The evolution of average temperature with time in the ROI is reported in figure 4b showing that temperature is increasing from the very beginning of the test. However, it must be noticed that the temperature increases along a time range (about $6 \mathrm{~ms}$ ) much longer than the duration of mechanical loading $(<1 \mathrm{~ms})$. 

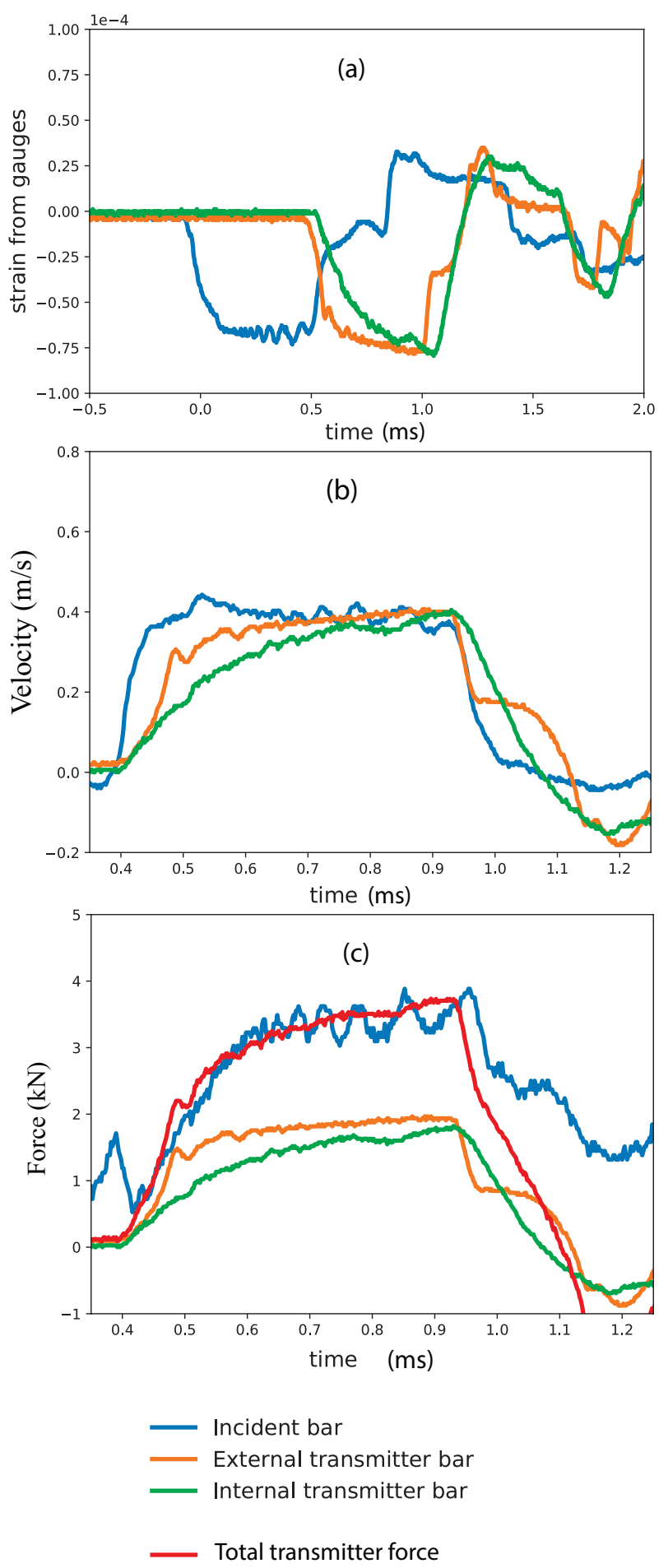

Fig. 3. Measurements from bars: (a) strain from gauges, (b) velocities, and (c) forces in bars.

\section{Modeling}

The numerical model used to describe the evolution of thermochemical fields is based on four scales: the variant scale $\alpha$ variant of a phase $\phi$ (martensite, $\mathrm{R}$ phase or austenite variants), the crystal $g$, the representative volume element (RVE) which is an assembly of crystals, and that of the structure. The single crystal model [7] predicts the distribution of the volume fractions $f_{\phi^{\alpha}}$ of each variant in
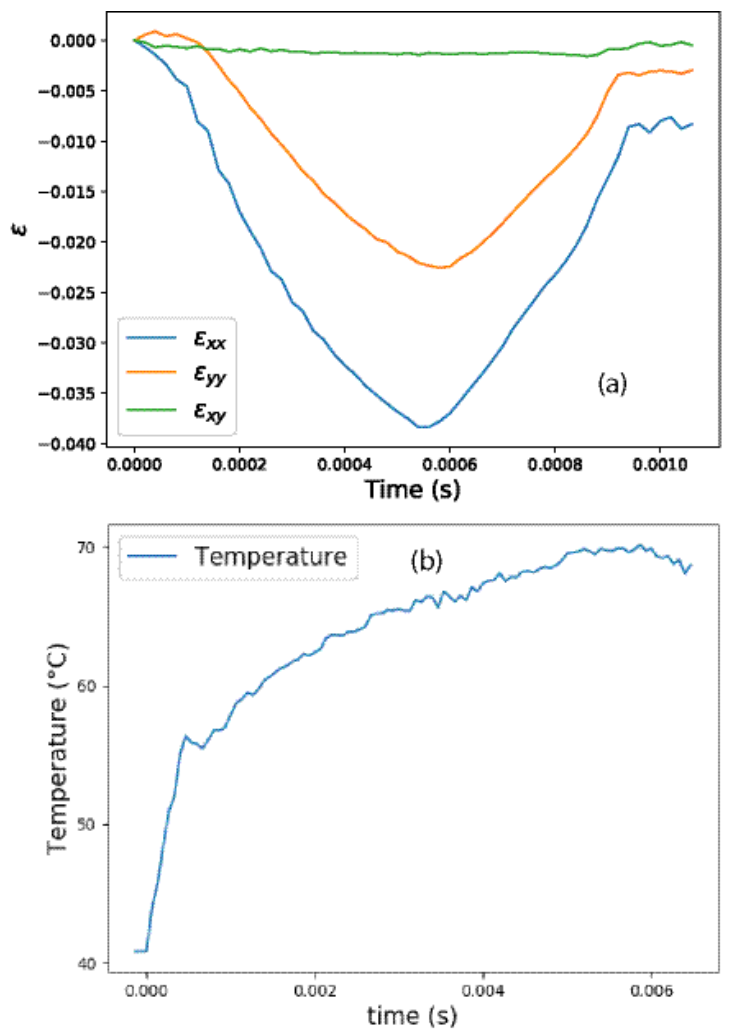

Fig. 4. Average strain components and temperature over the ROI from DIC and infrared observations.

the grain from the calculation of their Gibbs free energy $W_{\phi^{\alpha}}$ (3). The Gibbs free energy is the sum of chemical and elastic energies $(4,5)$, calculated from the knowledge of the enthalpies and entropies of each phase $\left(h_{\phi}, s_{\phi}\right)$, of the transformation strain (Green-Lagrange tensor) of each of variants $\boldsymbol{\epsilon}_{\phi^{\alpha}}^{t r}$ (12 variants of monoclinic martensite, 4 variants of rhombohedral $\mathrm{R}$ phase and 1 variant of cubic austenite), of the stiffness tensor of the medium $\mathbb{C}_{\phi}$, the temperature $T_{\phi^{\alpha}}$ and the stress $\sigma_{\phi^{\alpha}}$ (considered as homogeneous in the RVE to fasten the calculations). The fraction of each phase is estimated via a Boltzmann probability distribution (6), often used in magneto-mechanical problems [8], where $A_{s}$ is a parameter identified from a calorimetry measurement.

$$
\begin{gathered}
W_{\phi^{\alpha}}=W_{\phi^{\alpha}}^{T}+W_{\phi^{\alpha}}^{\sigma} \\
W_{\phi^{\alpha}}^{T}=h_{\phi}-T_{\phi^{\alpha}} S_{\phi} \\
W_{\phi^{\alpha}}^{\sigma}=-\frac{1}{2} \sigma_{\phi^{\alpha}}: \mathbb{C}_{\phi}^{-1}: \sigma_{\phi^{\alpha}}-\sigma_{\phi^{\alpha}}: \boldsymbol{\epsilon}_{\phi^{\alpha}}^{t r} \\
f_{\phi^{\alpha}}=\frac{\exp \left(-A_{s} W_{\phi^{\alpha}}\right)}{\sum_{\phi=1}^{n} \sum_{\alpha=1}^{m} \exp \left(-A_{s} W_{\phi^{\alpha}}\right)}
\end{gathered}
$$

The modeling of the polycrystal behavior is based on the scaling up by simple averaging of the quantities by crystal (using localization procedures if need be). For this, the RVE is described as an aggregate of single crystals via an Orientation Distribution Function (ODF). The RVE is composed of about 100 orientations for the calculation. 
The physical parameters used for the modeling are gathered in 1. A Backward Euler Finite Difference decomposition of the sample has been done in axisymmetic condition using Neumann Boundary condition (adiabatic condition), where phase fraction ratio acts as heat sources in the heat equation (7) [1].

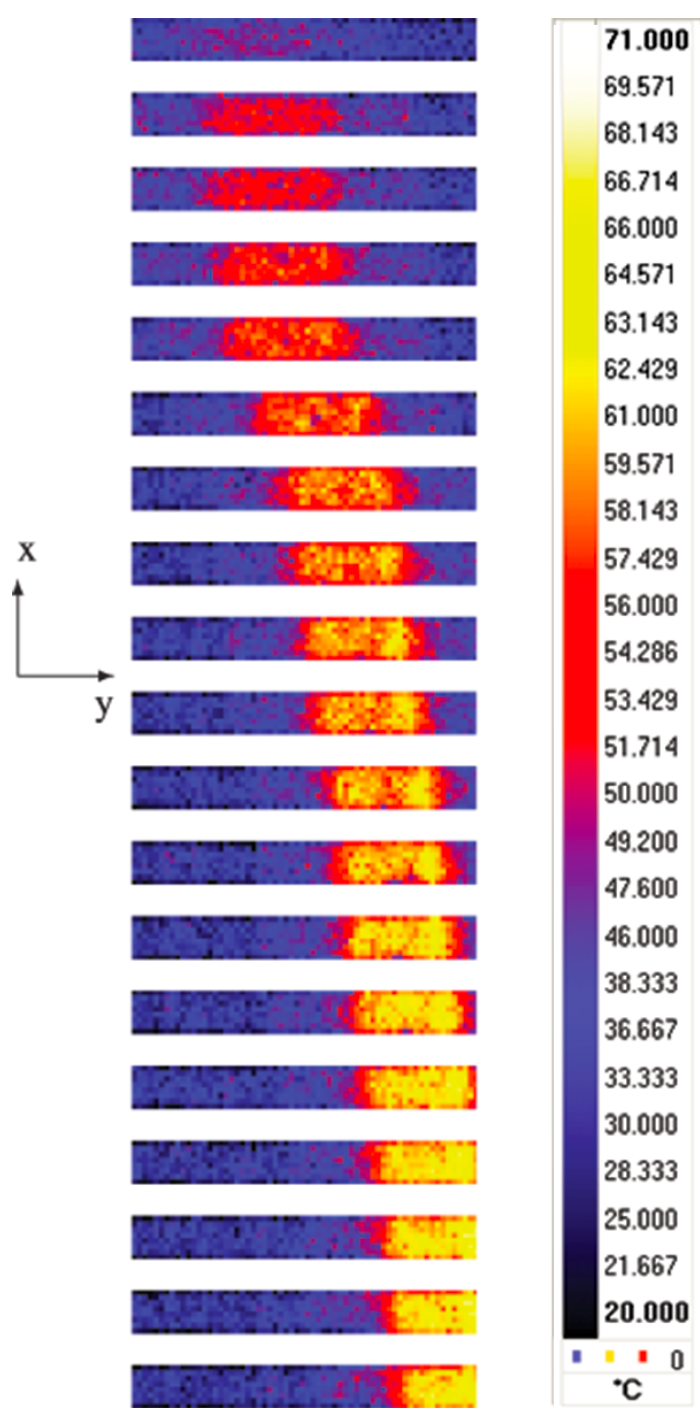

Fig. 5. Sequence of frames from infrared camera (6 ms).

$\rho C_{p} \frac{d T}{d t}=k \frac{d^{2} T}{d r^{2}}+\frac{k}{r} \frac{d T}{d r}-\left(h_{M}-h_{R}\right) \frac{d f_{M}}{d t}+\left(h_{R}-h_{A}\right) \frac{d f_{A}}{d t}$

Using the numerical discretization :

$$
\begin{gathered}
\frac{d T}{d t}=\frac{T_{j}^{n+1}-T_{j}^{n}}{\Delta t} \\
\frac{d f_{M}}{d t}=\frac{f_{M_{j}^{n+1}-f_{M}^{n}}^{n}}{\Delta t} \text { and } \frac{d f_{A}}{d t}=\frac{f_{A}^{n+1}-f_{A}^{n}}{\Delta t} \\
\frac{d^{2} T}{d r^{2}}=\frac{T_{j+1}^{n+1}-2 T_{j}^{n+1}+T_{j-1}^{n+1}}{\Delta r^{2}} \\
\frac{d T}{d r}=\frac{T_{j+1}^{n+1}-T_{j}^{n+1}}{\Delta r^{2}}
\end{gathered}
$$

A scalar representation of experimental and modeled stress-strain behaviors has been plotted in figure 6a (using a von Mises and Levy-Mises standardization for stress and strain. eq.(12) and (13)). We observe first in figure 6a that the threshold from elastic to inelastic transition (phase transition characterized by a plateau) seems underestimated by the modeling. This difference may be explained by the existence of some micro-inertia phenomena associated with the phase transition (rotation of crystallographic lattice) and ignored by the modeling. This underestimation happens although the temperature increase is overestimated at the very beginning of the loading (figure 6b). Indeed, the experimental temperature emission is spread over a much longer duration than the emission duration provided by the model, in direct relation with the duration of the shock. This difference may be explained by the existence of incubation delay associated with the germination of martensite in austenite. This phenomenon is ignored by the modeling. Despite this discrepancy, experimental and modeled final temperatures are in accordance, validating the adiabatic thermal boundary conditions.

$$
\begin{array}{r}
\sigma_{V M}=\sqrt{\frac{1}{2}\left(\left(\sigma_{x x}-\sigma_{y y}\right)^{2}+\sigma_{x x}^{2}+\sigma_{y y}^{2}\right)} \\
\epsilon_{L V}=\sqrt{\frac{2}{3}\left(\epsilon_{x x}^{2}+\epsilon_{y y}^{2}+2 \epsilon_{x y}^{2}\right)}
\end{array}
$$

\begin{tabular}{|c|c|c|c|c|c|c|}
\hline & $\begin{array}{c}h_{\phi} \\
\left(\mathrm{MJ} / \mathrm{m}^{3}\right)\end{array}$ & \multicolumn{2}{|c|}{$\begin{array}{l}s_{\phi}(273 \mathrm{~K}) \\
\left(\mathrm{MJ} / \mathrm{m}^{3}\right)\end{array}$} & \multicolumn{3}{|c|}{$\begin{array}{l}\text { lattice parameters } \\
\qquad(\mathrm{nm})\end{array}$} \\
\hline A & -110 & \multicolumn{2}{|c|}{0.72} & \multicolumn{3}{|c|}{$\mathrm{a}=\mathrm{b}=\mathrm{c}=0.3017$} \\
\hline $\mathrm{R}$ & -254 & \multicolumn{2}{|c|}{0.57} & \multicolumn{3}{|c|}{$a=b=0.734 c=0.527$} \\
\hline M & -293 & \multicolumn{2}{|c|}{0.20} & \multicolumn{3}{|c|}{$a=0.290 b=0.411 c=0.465$} \\
\hline & \multicolumn{2}{|c|}{$\begin{array}{c}\text { lattice angles } \\
\left({ }^{\circ}\right)\end{array}$} & \multicolumn{4}{|c|}{$\begin{array}{c}C_{i j} \text { (Voigt) } \\
(\mathrm{GPa})\end{array}$} \\
\hline $\mathrm{A}$ & \multirow{3}{*}{\multicolumn{2}{|c|}{$\begin{array}{c}\alpha=\beta=\gamma=90 \\
\alpha=\gamma=90, \beta=120 \\
\alpha=\gamma=90, \beta=97.8\end{array}$}} & \multirow{3}{*}{\multicolumn{4}{|c|}{$C_{11}=238, C_{12}=142, C_{44}=232$}} \\
\hline $\mathrm{R}$ & & & & & & \\
\hline M & & & & & & \\
\hline & \multicolumn{2}{|c|}{$\begin{array}{c}\rho \\
\left(\mathrm{kg} / \mathrm{m}^{3}\right)\end{array}$} & \multicolumn{2}{|c|}{$\begin{array}{c}C_{p} \\
(\mathrm{~J} / \mathrm{kg} / \mathrm{K})\end{array}$} & $\begin{array}{c}A_{s} \\
\left(\mathrm{~m}^{3} / \mathrm{J}\right)\end{array}$ & \\
\hline & \multicolumn{2}{|c|}{6450} & \multicolumn{2}{|c|}{900} & $2.54 \mathrm{e}-06$ & \\
\hline
\end{tabular}

Table 1. Physical constants used for the multiscale modeling.

\section{Conclusion}

In this study, the multi-axial dynamic behavior of a NiTi alloy has been prospected and compared to the results of axisymmetric finite difference modeling including a multiscale and multiphysic constitutive law. Despite very strong assumption considering the mechanical and thermal boundary conditions, interesting qualitative results have been obtained. Improvements may be achieved by more representative boundary conditions, the introduction of micro- inertial phenomena in the modeling, and a time delay in martensite production able to model the heat emission more accurately. 

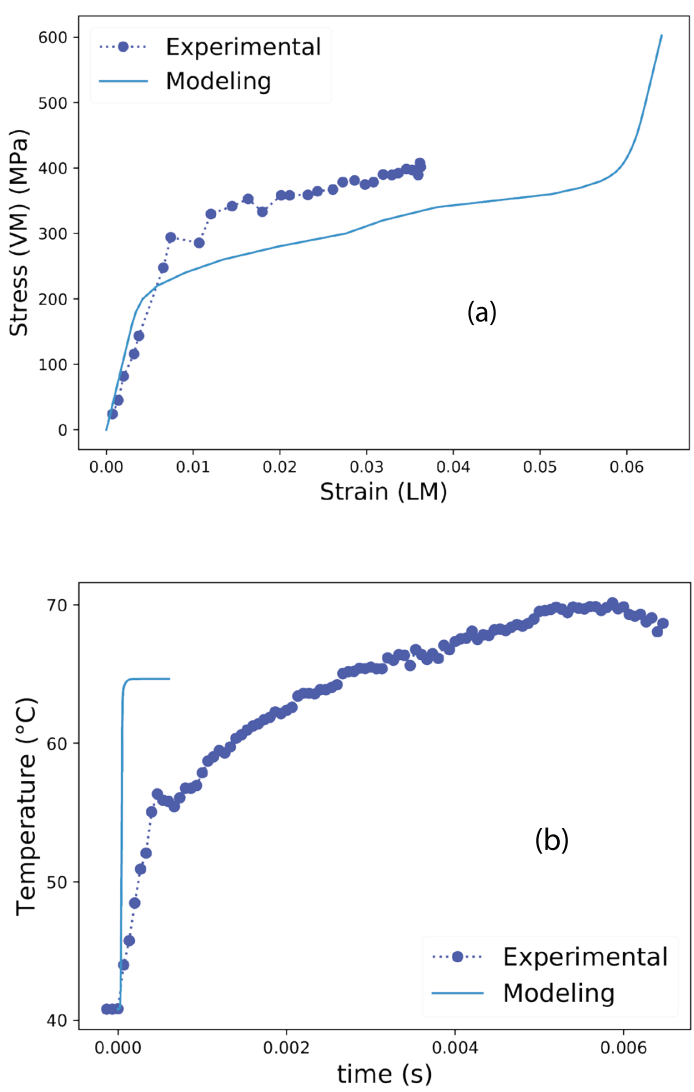

Fig. 6. Comparison experiments modeling: (a) Normalized stress-strain behavior; (b) Temperature release.

\section{References}

1. D. Depriester, A. Maynadier, K. Lavernhe-Taillard, O. Hubert, "Thermomechanical modelling of a NiTi SMA sample submitted to displacement-controlled tensile test" International Journal of Solids and Structures, 51, 10, 1901-1922 (2014)

2. R. Adharapurapu, F. Jiang, S.Vecchio and G. T. Gray, Response of NiTi shape memory alloy at high strain rate: A systematic investigation of temperature effects on tension-compression asymmetry Acta Materialia (2006)

3. B. Hopkinson. A method of measuring the pressure produced in the detonation of high explosives or by the impact of bullets, Philosophical Transactions of the Royal Society of London, 213, 437-456 (1914)

4. Z. Tomicevc, F. Hild, S. Roux. Mechanics-aided digital image correlation. Journal of Strain Analysis, 48(5), 330-343 (2013)

5. E.Davies and S. Hunter. The dynamic com- pression testing of solids by the method of the split hopkinson pressure bar. Journal of the Mechanics and Physics of Solids (1963)

6. H.Zhao and G.Gary. On the use of SHPB technique to determine the dynamic behavior of the materials in the range of small strains. International Journal of Solids and structures (1996)

7. A. Maynadier, D. Depriester, K. Lavernhe-Taillard and O.Hubert, "Thermo-mechanical description of phase transformation in Ni-Ti Shape Memory Alloy", Procedia Engineering, 10 2214-2219 (2011)

8. X. Wang, O. Hubert, S. He, F.S. Mballa-Mballa, Y. Huang, "Reversible magneto-mechanical modeling of heterogeneous media", ournal of Superconductivity and Novel Magnetism, 27, 9, 2049-2058 (2014) 
